\title{
El fantasma de la perspectiva de género. Aportes teóricos y metodológicos para el análisis del manejo de los recursos naturales
}

\section{The specter of the gender perspective: theoretical and methodologycal contribution for the analysis of natural resource use}

\author{
Verónica Vázquez GARCÍA, María de las Mercedes ROCHA GÓMEZ, \\ Elda Montero MENDOZA
}

\begin{abstract}
RESUMEN
Este artículo discute la contribución de la perspectiva de género a la Teoría de Sistemas Agrícolas o Farming Systems Theory (FST), la cual se dedica al estudio de las actividades productivas realizadas por el grupo doméstico y propone la integración de los productores en el proceso de investigación. La relevancia de la perspectiva de género para la FST se centra en tres puntos principales. Primero, recalca la importancia de tomar en cuenta en el análisis al trabajo reproductivo, la producción casera de objetos para uso doméstico y para la venta y la generación de ingresos en actividades no relacionadas con la producción agrícola, generalmente a cargo de las mujeres. Segundo, propone ver al grupo doméstico como un espacio de conflicto y negociación, donde hombres y mujeres tienen responsabilidades diferentes, acceso a distintos recursos y un control diferenciado sobre los ingresos y el producto de su trabajo. Tercero, sugiere que nos aseguremos de que el proceso de investigación incorpore a todos y todas las integrantes del grupo doméstico, es decir, a hombres y mujeres. Sólo así podemos hablar de un proceso realmente participativo de extensionismo rural. El texto utiliza ejemplos del campo mexicano para ilustrar el análisis.
\end{abstract}

\begin{abstract}
This paper discusses the contribution of gender analysis to Farming Systems Theory (FST), whish focuses on the productive activities of households and argues that producers must be involved in the research process. The relevance of gender analysis for FST can be summarized in three major points. First. FST must take into account reproductive activities; household production of goods for domestic use or sale and other forms of income generating activities, activities which are usually carried out by women. Second, the household must be seen as an arena of conflict and negotiation, where men and women have different responsibilities, as well as different access and control over income and resources. Third, researchers have to make sure that all members of the household participate in the research process. Only then can we speak of participatory rural extensionism. The paper uses examples of the Mexican countryside to illustrate the argument
\end{abstract}




\section{Introducción}

"Un fantasma recorre al mundo" desde hace algunas décadas, transformando profundamente la forma de hacer ciencia y política pública. Este fantasma, que no se logra ver claramente pero que está presente en aulas de universidades, cubículos de investigación, oficinas y pasillos de gobierno y de agencias internacionales, es la perspectiva de género.

En este artículo nos proponemos abordar la contribución de la perspectiva de género a la Teoría de Sistemas Agrícolas. Dividiremos nuestra exposición en tres partes. En primera instancia hablaremos brevemente sobre los aportes de la perspectiva de género al concepto de desarrollo sustentable. En la segunda discutiremos la literatura de género y medio ambiente para abordar en la tercera y última sección los principios básicos y la metodología de trabajo de la Teoría de Sistemas Agrícolas (FST por sus siglas en inglés). Este recorrido nos permitirá comprender desde diversos ángulos la problemática del manejo de recursos naturales con perspectiva de género.

\section{La perspectiva de género y el concepto de desarrollo sustentable}

Como cuerpo teórico, la perspectiva de género se propone explorar los procesos de diferenciación, dominación y subordinación entre hombres y mujeres. Según Daniel Cazés (1998, p. 39), las cuestiones sustantivas que se plantean desde la perspectiva de género son:

¿En qué medida la organización patriarcal del mundo facilita o impide a las mujeres y hombres satisfacer sus necesidades vitales y realizar sus aspiraciones?

¿Cuál es la distancia y la relación entre el desarrollo personal y social de las mujeres y el de los hombres?

¿De qué manera inciden las relaciones de dominio y opresión entre los géneros, en el desarrollo social, el ejercicio de los derechos humanos y la calidad de vida de hombres y mujeres?
¿Cómo se desarrolla históricamente la desigualdad genérica, y qué alternativas hay frente a ésta?

Una de las categorías más importantes dentro de esta perspectiva es la de género, la cual parte del siguiente planteamiento: las características humanas consideradas como "femeninas" son adquiridas mediante la socialización, en lugar de derivarse "naturalmente" del sexo o de la condición biológica de las mujeres. Al decir que las mujeres no nacen, sino se hacen, Simone de Beauvoir hace la primera declaración célebre sobre el género. Posteriormente la categoría es retomada por feministas anglosajonas para hacer estudios sobre identidad sexual. Actualmente, la categoría género está "en el centro de uno de los debates políticos más trascendentes: el del papel de las mujeres en la sociedad" (Marta Lamas, 1996, p. 9-11).

La categoría de género abre entonces la posibilidad de cambiar los papeles y atributos asignados a hombres y mujeres, al decir que no son naturales sino construidos socialmente. De esta manera, la perspectiva de género "se aleja de las argumentaciones funcionalistas y deterministas, y busca explicar la acción humana como un producto construido con base en un sentido subjetivo" (Lamas, 1996, p. 11). En pocas palabras, dicha perspectiva tiene un profundo potencial transformador de la sociedad.

La teoría feminista ha contribuido prácticamente a todos los debates de las ciencias sociales desarrollados en las últimas tres décadas. El problema de la sustentabilidad no es la excepción. Parte central de este debate es precisamente qué tipo de sociedad hay que tener para satisfacer nuestras necesidades sin destruir la naturaleza. En otras palabras, nos preguntamos para qué queremos conservar los recursos y qué papel juegan los seres humanos en dicha conservación.

Esta pregunta ha sido respondida desde una gran variedad de perspectivas. En este trabajo confrontaremos las ideas de la teoría económica dominante, que se ha convertido en política pública de corte neo-liberal en la mayoría de los países del mundo, con las ideas de algunas feministas que han reflexionado sobre el tema. 
Para la postura neo-liberal, la conservación de los recursos naturales permite generar riqueza y sostener el ritmo de crecimiento, por lo que es de utilidad para el progreso económico (Barrón y de Remes, 1996, p. 14; Gudynas, 1992, p. 112). En este sentido, conservación y desarrollo sustentable van de la mano, puesto que se proponen incentivar la producción que no contamina ni depreda y aplicar impuestos a la que sí lo hace ${ }^{1}$. Se argumenta que al pasar de un sistema de prohibiciones a uno de incentivos se producirá más limpiamente y las personas valorarán los recursos naturales, porque éstos tendrán un valor monetario (Barrón y de Remes, 1996, p. 18).

La perspectiva de género tiene una postura distinta. Sostiene que los recursos naturales no tienen únicamente un valor económico, sino que también ofrecen beneficios de naturaleza cualitativa (Warring, 1993, p. 50). En este sentido, el enfoque de género es crítico del modelo de desarrollo basado en el postulado del progreso económico ya que, como lo señala Kamila Bashin (1993), el crecimiento del Producto Interno Bruto (PIB) en realidad no mide el bienestar de la gente. En las dos últimas décadas el PIB de muchos países latinoamericanos se ha mantenido a la alta ${ }^{2}$, pero lo mismo ha sucedido con la población pobre: sigue aumentando.

Otra diferencia notable en el concepto de desarrollo sustentable del neo-liberalismo y de la perspectiva de género tiene que ver con la definición de los agentes de cambio. Para los neo-liberales, la participación económica del Estado debe ser mínima. Los actores que intervendrán para resolver la crisis ecológica son el mercado internacional y el libre comercio. Los inversionistas y el gobierno que los apoya estarán a cargo de tomar las medidas concretas, y desde su perspectiva es prioritario privatizar los recursos naturales (Gudynas, 1992; Leis, 1992; Jaakonkari, 1996).

El punto importante en esta discusión es, ¿qué papel jugamos los y las ciudadanas que no tenemos acceso a las esferas de decisión ni posibilidades de "comprar" recursos naturales? ¿Significa algo para los y las pobres este cambio en la distribución de los recursos naturales? La perspectiva de género tiene una respuesta a estas interrogantes. Parte de una crítica del desarrollo y de las formas de organización social que han predominado hasta nuestros días, $y$ pone énfasis en una mayor participación de la gente, especialmente de los grupos marginados (Bhasin, 1993; Häusler, 1994; Lagarde, 1997; Warring, 1993). Esta perspectiva sostiene que la sociedad civil debe ser la protagonista del cambio, con el fin de lograr el objetivo de la equidad entre géneros, razas, clases y generaciones. En esto consiste el desarrollo sustentable.

Marylin Warring (1993), economista neozelandesa, tiene una propuesta concreta sobre el manejo del Sistema de Cuentas Nacionales desde una perspectiva de género. Ella propone medir no sólo el trabajo remunerado, sino también el impacto de determinadas actividades productivas (por ejemplo la industria militar, que genera enormes beneficios económicos) en los ecosistemas, la calidad de vida y el bienestar de la población. Es decir, el PIB no es un indicador suficiente sobre la situación de los y las habitantes de un país. Además, dicho sistema debe visibilizar el trabajo de las mujeres, particularmente el llamado doméstico o reproductivo, que es indispen-

\footnotetext{
'Quienes usen tecnologías eficientes y limpias serán alentados sin impuestos adicionales, así sus productos tendrán un costo menor. Mientras que los productos que desperdician o dañan el ambiente deben ser más caros, aplicándoseles un impuesto.

2 Según cifras de la CEPAL, el PIB per cápita de México en 1989 era de 3157 dólares americanos. En la década de los noventa no aumentó mucho pero se mantuvo. Para 1994 había experimentado un ligero incremento a 3386 dólares, mientras que en 1997 era de 3394 dólares. Si bien no existe un acuerdo sobre cómo medir la pobreza, las cifras aportadas por la misma CEPAL indican un incremento del porcentaje de hogares que se encuentran por debajo de la línea de pobreza: en 1989 era de $39 \%$ y en 1996 alcanza el $43 \%$ (http://www.eclac.cl). Para el año 2000 la CEPAL proyecta un aumento porcentual de 4.5 en el PIB con respecto al año anterior, lo cual no representa una mejoría significativa (González Amador, La Jornada, 17 enero 2000). Al mismo tiempo, la pobreza en México preocupa hasta al Banco Mundial, el cual consideró en un reporte especial sobre México que la pobreza en el país es un problema "extendido y persistente". Calificó de "preocupante" el que la proporción de mexicanos(as) que viven en la pobreza se haya incrementado sostenidamente desde finales de los ochenta, "a pesar del rápido crecimiento económico" (Márquez Ayala, La Jornada, 19 septiembre 1999).
} 
sable para la sobrevivencia del grupo doméstico en particular, y de la humanidad en general. Es una jornada $\sin$ fin que no es valorada puesto que no se genera ningún ingreso con ella. Sabemos que los censos de su propio país y el canadiense han empezado a dar los primeros pasos en esta dirección.

Para concluir esta sección, diremos entonces que el desarrollo sustentable para el neoliberalismo es la "aplicación de políticas ambientales eficientes y compatibles con el crecimiento económico en el largo plazo, basadas en su mayoría en el cambio de la estructura de incentivos... para crecer $y$ conservar" (Barrón y de Remes, 1996, p. 15). Esta definición no contempla la superación de las diferencias sociales, ni cuestiona las relaciones de poder o los niveles de consumo (y desperdicio) de los ricos. Por su parte, la teoría de género propone que cuestionemos los indicadores que miden únicamente el "desempeño" de la economía y nos enfoquemos en otros de tipo social. El desarrollo sustentable debe preocuparse por el presente y el futuro, establecer relaciones de armonía con la naturaleza, centrarse en la persona, orientarse hacia las necesidades básicas, traer consigo la descentralización del poder y por lo tanto estar basado en la democracia directa, y promover una política de paz y de respeto por la vida. En pocas palabras, debe de darse de abajo hacia arriba (Bhasin, 1993), lo que implica no sólo un cambio en las relaciones de poder, sino en el modelo de desarrollo dominante.

\section{De Mujeres y Medio Ambiente (MMA) a Género y Medio Ambiente (GMA)}

En esta parte del trabajo analizaremos diversas corrientes de pensamiento elaboradas por académicas feministas de todo el mundo, que a partir de la década de los setenta se propusieron estudiar y dar a conocer el papel que juegan las mujeres en el desarrollo, particularmente en países del llamado Tercer Mundo. Nos centraremos en las corrientes de Mujeres y Medio Ambiente y Género y Medio Ambiente.

La corriente Mujeres y Medio Ambiente (MMA) tiene como punto de partida la publicación del libro de Esther Boserup (1970), El papel de las mujeres en el desarrollo económico. En éste, la autora demuestra que la introducción de tecnologías agrícolas ha tenido un efecto negativo en la vida de las mujeres rurales de Africa, al cambiar la división genérica del trabajo y desplazarlas de áreas tradicionales de influencia femenina (Braidotti, 1997, p. 79). A partir de dicha publicación, las especialistas comienzan a preguntarse sobre el significado del desarrollo para las mujeres, desde algunas disciplinas relacionadas con el medio ambiente, como son la silvicultura y la agricultura. La perspectiva de MMA fundamenta su análisis en los roles de mujeres y hombres en la división genérica del trabajo. Por ejemplo, analiza el papel de las mujeres rurales en la recolección de leña y en el aprovisionamiento de agua; el papel de las mujeres como consumidoras de productos de limpieza en zonas urbanas; etcétera (ver Rodda, 1993, para más ejemplos).

Un problema en esta perspectiva ha sido el responsabilizar a las mujeres de la crisis ambiental del planeta. Si las mujeres son las recolectoras de los recursos naturales, también son ellas quienes están devastando el ambiente. En los años ochenta se pensaba que el uso de leña para consumo doméstico era la causa de la deforestación en países del Sur. En esta época fue popular la imagen de los pobres y particularmente de las mujeres destruyendo su propio medio ambiente. Surgieron entonces programas tendientes a disminuir el consumo de leña con estufas ahorradoras, pero los mismos técnicos y técnicas que las promovían se dieron cuenta que la escasez leña se debía a la tala inmoderada a gran escala (Braidotti, 1994, p. 84).

Es a partir de la corriente llamada Género y Medio Ambiente (GMA) que estas percepciones son rectificadas. Dicha corriente parte de considerar las relaciones sociales que establecen las mujeres con el sistema del poder en el que están insertas. Se les deja de ver en forma homogénea y se reconoce que no todas las mujeres experimentan la degradación ambiental de igual forma. Al mismo tiempo, se reconoce que los problemas ambientales impactan de diferentes formas a los grupos de mujeres según su edad, clase social, etnia, etcétera (Rico, 1997). 
Otra característica de la corriente GMA es cuestionar la asociación crecimiento demográficopobreza-devastación del ambiente, basada en la teoría Malthusiana cuya tesis central es que la población aumenta en progresión geométrica, mientras que la oferta de alimentos lo hace en progresión aritmética. De esta manera, el crecimiento demográfico es visto como la causa de la pobreza y el deterioro ambiental. Se asume entonces que las mujeres, en su papel de reproductoras biológicas de la sociedad, pueden contribuir a reducir la tasa de crecimiento poblacional, con lo cual automáticamente se reduce la pobreza y se protege al medio ambiente (Careaga et al., 1997, p. 18-19).

Esta tesis ha sido tachada de reduccionista y simplista, ya que no toma en cuenta "las distintas formas en que los grupos humanos han ocupado $\mathrm{y}$ manejado ecosistemas particulares" (Careaga et al., 1997, p. 6). Bajo el enfoque de GMA se demuestra que si bien la sobrepoblación tiene consecuencias sobre el medio ambiente, también hay que considerar las diferencias en consumo entre la gran masa de pobres del mundo y los pocos ricos de países desarrollados y del Tercer Mundo. María Mies (1992, p. 56) señala que un quinto de la población mundial (que vive en los países industrializados), consume el $85 \%$ de los recursos del planeta y produce el $80 \%$ del total de los desperdicios y contaminantes.

El tipo de proyectos de desarrollo que son propuestos y promovidos también marca una diferencia importante entre las corrientes de MMA y GMA. Desde la primera, se impulsan proyectos que no representan los intereses de las mujeres sino más bien aumentan su carga de trabajo (Braidotti, 1994, p. 98). Ejemplos de éstos son: construcción de terrazas para la conservación del suelo, cuidado de semillas y hierbas para salvaguardar la biodiversidad, plantación de árboles. Además de cuidar hijas e hijos, ancianas y ancianos, ahora las mujeres también son responsables de cuidar a la naturaleza, pero el beneficio que derivan de ello no está claro para nadie. Las mujeres son vistas como simple fuerza de trabajo o, en palabras de Leach et al. (1997), como "agentes capaces que pueden ser movilizados en proyectos de conservación", pero no como sujetas de su propio desarrollo.

Por su parte, la corriente de GMA reconoce que tanto mujeres como hombres son capaces de cuidar el ambiente, por lo que ambos deben contribuir en la elaboración de diagnósticos y planes de acción. Rico (1997) argumenta que todos los indicadores deben de ser desagregados por sexo, de forma que podamos ilustrar y documentar el sistema desigual que regula el trabajo y el acceso a recursos por parte de mujeres y hombres. Igualmente, Leach et al. (1997) proponen que para identificar diferencias entre grupos de mujeres y entre ambos sexos es necesario hacer "una desestructuración detallada de diferencias y divisiones en actividades, responsabilidades y derechos en procesos de uso y administración de recursos naturales" por parte de mujeres y hombres. En otras palabras, se pretende estudiar las estructuras de poder y los procesos identitarios que regulan el acceso de mujeres y hombres a determinados recursos, las relaciones de trabajo que establecen entre ellas y ellos, y el control y beneficios que derivan de su trabajo. Enseguida ilustraremos estas ideas utilizando como ejemplo la Farming Systems Theory, o Teoría de los Sistemas Agrícolas.

\section{Los sistemas agrícolas desde una perspectiva de género: Farming Systems Theory (FST)}

No es fácil establecer con exactitud en qué consiste FST, puesto que una gran variedad de trabajos han sido producidos bajo esta óptica. Sin embargo, sí pueden establecerse algunos principios básicos de los que parten estos estudios. Uno de ellos es que la mayor parte de los grupos domésticos en países subdesarrollados cuentan con pocos recursos para producir, además del trabajo familiar no pagado. Sus actividades son complejas y multifacéticas, y están dirigidas no sólo a la subsistencia sino también a la producción para el mercado. En este sentido, Susan Poats et al. (1988, p. 2) establecen que FST tiene las siguientes características:

ISe propone trabajar con productores y productoras de pocos recursos, que a pesar de ser 
una parte importante de la población rural en la mayoría de los países del Tercer Mundo, son pocas las políticas públicas orientadas a apoyar de manera eficiente y equitativa sus actividades productivas.

IToma un enfoque sistémico, el cual permite examinar la complejidad de sus actividades. Es decir, FST pretende incluir en el análisis la gran diversidad de labores realizadas por los y las integrantes del grupo doméstico para su subsistencia.

ICentra su análisis en el grupo doméstico, es decir, en las personas que comparten la misma mesa y el mismo techo, y pueden tener lazos de parentesco entre sí. Estas personas establecen relaciones de trabajo entre sí y para con el medio ambiente.

Incluye a los y las productoras en el proceso de investigación, desde el planteamiento inicial del problema hasta las recomendaciones finales.

Por su parte, Shaner et al. (1981, p. 18) señalan que FST se concentra en dos áreas principales:

-La relación entre las diferentes partes del sistema agrícola, que son manejadas por los y las integrantes del grupo doméstico. En el contexto indígena mexicano, estas partes son la milpa, visto como un espacio de reproducción física y cultural de los y las campesinas; el acahual, o las tierras en descanso; el solar, donde se tienen frutales, hierbas de olor y animales domésticos, los cuales apoyan en gran medida la dieta familiar; y el monte, de donde se extraen recursos maderables y no maderables para diversos usos (leña, construcción, alimento, etcétera).

$\triangle \mathrm{La}$ forma en que dichas partes se relacionan con factores físicos, biológicos y socioeconómicos ajenos a los y las integrantes del grupo doméstico. Estos factores pueden ser el clima, el tipo de ecosistema en el que se habita, las ofertas de trabajo remunerado en la zona, los niveles de migración, etcétera.

La FST tiene una metodología concreta de trabajo, que ha sido sistematizada por Alistair
Sutherland (1987) utilizando material del taller El papel de la sociología rural y de la antropología en la investigación y el extensionismo de FS, organizado por CIMMYT. Dicha metodología comprende cuatro etapas:

La elaboración del diagnóstico, cuyo propósito es entender las prioridades del grupo doméstico, la forma en que manejan sus sistemas agrícolas, cómo deciden utilizar los recursos naturales a su disposición y responder al contexto económico en el que están ubicados.

-La planeación del trabajo que se va a realizar. En esta etapa se deben identificar técnicas y materiales que pueden ayudar a resolver los problemas de los sistemas en cuestión. Hay que asegurarse de incorporar a los integrantes del grupo doméstico en la búsqueda de soluciones, enfocarse no sólo en elementos técnicos sino también en los procesos sociales que acompañan la adopción de nuevas tecnologías y nuevas formas de hacer las cosas. ILa experimentación y evaluación, etapa donde se implementan y evalúan las soluciones propuestas en colaboración con los integrantes de la comunidad.

IRecomendaciones y extensionismo, etapa en la que se sistematiza el trabajo realizado y se elaboran recomendaciones para futuros trabajos.

Algunas especialistas que trabajan el tema de género y medio ambiente han recurrido a los principios y metodología de FST debido a su énfasis en el grupo doméstico, la agricultura de escasos recursos y la participación de productores y productoras en la investigación. Estos postulados han sido parte importante de los estudios de género en el medio rural, incluso desde antes de que se formulara en forma explícita la postura teórica y la metodología de FST. Sin embargo, ha sido necesario transformar al FST para adecuarlo a la perspectiva de género en dos líneas principales, las cuales se exponen a continuación.

Primero, fue indispensable incluir dentro del análisis al trabajo reproductivo, la producción casera 
una parte importante de la población rural en la mayoría de los países del Tercer Mundo, son pocas las políticas públicas orientadas a apoyar de manera eficiente y equitativa sus actividades productivas.

IToma un enfoque sistémico, el cual permite examinar la complejidad de sus actividades. Es decir, FST pretende incluir en el análisis la gran diversidad de labores realizadas por los y las integrantes del grupo doméstico para su subsistencia.

ICentra su análisis en el grupo doméstico, es decir, en las personas que comparten la misma mesa y el mismo techo, y pueden tener lazos de parentesco entre sí. Estas personas establecen relaciones de trabajo entre sí y para con el medio ambiente.

Incluye a los y las productoras en el proceso de investigación, desde el planteamiento inicial del problema hasta las recomendaciones finales.

Por su parte, Shaner et al. $(1981$, p. 18) señalan que FST se concentra en dos áreas principales:

La relación entre las diferentes partes del sistema agrícola, que son manejadas por los y las integrantes del grupo doméstico. En el contexto indígena mexicano, estas partes son la milpa, visto como un espacio de reproducción física y cultural de los y las campesinas; el acahual, o las tierras en descanso; el solar, donde se tienen frutales, hierbas de olor y animales domésticos, los cuales apoyan en gran medida la dieta familiar; y el monte, de donde se extraen recursos maderables y no maderables para diversos usos (leña, construcción, alimento, etcétera). I La forma en que dichas partes se relacionan con factores físicos, biológicos y socioeconómicos ajenos a los y las integrantes del grupo doméstico. Estos factores pueden ser el clima, el tipo de ecosistema en el que se habita, las ofertas de trabajo remunerado en la zona, los niveles de migración, etcétera.

La FST tiene una metodología concreta de trabajo, que ha sido sistematizada por Alistair
Sutherland (1987) utilizando material del taller $E l$ papel de la sociología rural y de la antropología en la investigación y el extensionismo de FS, organizado por CIMMYT. Dicha metodología comprende cuatro etapas:

ILa elaboración del diagnóstico, cuyo propósito es entender las prioridades del grupo doméstico, la forma en que manejan sus sistemas agrícolas, cómo deciden utilizar los recursos naturales a su disposición y responder al contexto económico en el que están ubicados.

ILa planeación del trabajo que se va a realizar. En esta etapa se deben identificar técnicas y materiales que pueden ayudar a resolver los problemas de los sistemas en cuestión. Hay que asegurarse de incorporar a los integrantes del grupo doméstico en la búsqueda de soluciones, enfocarse no sólo en elementos técnicos sino también en los procesos sociales que acompañan la adopción de nuevas tecnologías y nuevas formas de hacer las cosas. I La experimentación y evaluación, etapa donde se implementan y evalúan las soluciones propuestas en colaboración con los integrantes de la comunidad.

-Recomendaciones y extensionismo, etapa en la que se sistematiza el trabajo realizado y se elaboran recomendaciones para futuros trabajos.

Algunas especialistas que trabajan el tema de género y medio ambiente han recurrido a los principios y metodología de FST debido a su énfasis en el grupo doméstico, la agricultura de escasos recursos y la participación de productores y productoras en la investigación. Estos postulados han sido parte importante de los estudios de género en el medio rural, incluso desde antes de que se formulara en forma explícita la postura teórica y la metodología de FST. Sin embargo, ha sido necesario transformar al FST para adecuarlo a la perspectiva de género en dos líneas principales, las cuales se exponen a continuación.

Primero, fue indispensable incluir dentro del análisis al trabajo reproductivo, la producción casera 
de objetos para uso doméstico y para la venta y la generación de ingresos en actividades no relacionadas con la producción agrícola. Además del análisis detallado de la división genérica del trabajo (qué actividades hacen las mujeres y qué actividades hacen los hombres en los distintos ámbitos productivos y reproductivos), es importante estudiar el valor que la misma comunidad le da a las actividades femeninas, el acceso (formal e informal) a recursos por parte de las mujeres, el control que ejercen sobre ellos, y los beneficios que derivan de su trabajo. Por ejemplo, en el México indígena es bien sabido que las fincas cafetaleras son cuidadas y trabajadas por todos los y las integrantes del grupo doméstico. Sin embargo, la tierra donde se encuentra la finca está generalmente a nombre del jefe de familia, tanto en términos legales como sociales, por lo que las mujeres tienen poco poder de decisión sobre el uso de la tierra. Igualmente, el café que hombres, mujeres, niños y niñas cortan a lo largo de varios meses es vendido por el mismo jefe de familia, el cual decide el destino de los ingresos producto de la venta. En pocas palabras, mujeres, niños y niñas trabajan en la finca cafetalera, pero tienen poco control sobre la tierra y derivan escasos beneficios de su trabajo (Vázquez García, en prensa).

Segundo, y en esta misma línea, es necesario cuestionar la idea de que el grupo doméstico es homogéneo y unitario y sus integrantes comparten intereses y recursos. La perspectiva de género concibe al grupo doméstico como un espacio de conflicto y negociación, donde hombres y mujeres tienen responsabilidades diferentes, acceso a distintos recursos y un control diferenciado sobre los ingresos y el producto de su trabajo. Es crucial tomar en cuenta estas diferencias en el análisis para no invisibilizar la desigualdad entre hombres y mujeres en el manejo de recursos naturales. Retomando el caso de la finca cafetalera en el México indígena, es necesario considerar las desigualdades en la distribución de los ingresos producto del corte de café, para realmente elaborar propuestas de trabajo que beneficien a las mujeres de la misma forma que a los hombres.

Retomando la metodología propuesta por Sutherland (1987), tendríamos que asegurarnos entonces de que nuestro diagnóstico incorpore a todos y todas las integrantes del grupo doméstico, ya que el trabajo que realizan los hombres y las mujeres no es el mismo, y por lo tanto los problemas que enfrentan tampoco son iguales. No podemos asumir que las mujeres realizan sólo labores domésticas y que no tienen nada que decir sobre los espacios productivos. Ya hemos visto que en el caso de la finca cafetalera indígena, el trabajo femenino no es reconocido ni recompensado en su verdadera dimensión. Asimismo, no podemos pensar que las decisiones sobre cuándo sembrar, cómo gastar el dinero de la cosecha, cuántos jornales contratar, etcétera, se toman sin que haya conflicto al interior del grupo doméstico, ya que estaríamos reproduciendo relaciones de desigualdad de género insertas en los procesos de trabajo y en la distribución de los beneficios. No podemos suponer que las mujeres se van a interesar en las mismas especies maderables propuestas en un taller al que sólo asistieron hombres, ya que ellas hacen uso distinto de la madera. Sería un error asumir que las mujeres que emigran a las ciudades para emplearse como trabajadoras domésticas lo hacen por gusto, y que sus ingresos no representan nada para el grupo doméstico.

En la etapa de la planeación es importante buscar soluciones a problemas no sólo en la parcela, sino también en la casa. En el campo mexicano la producción de la milpa comienza desde la selección de las semillas, que se hace en el solar, y termina con el almacenamiento de la mazorca según tipo y calidad, y con la elaboración de las tortillas, trabajo que hacen las mujeres. La tortilla hecha a mano sigue siendo el alimento principal de los y las campesinas de México y la milpa se hace principalmente para tener tortillas calientes en la mesa. Es importante ver el ciclo productivo completo y preguntarles a hombres y mujeres qué alternativas ven para fortalecerlo.

Finalmente, en las dos últimas etapas presentadas por Sutherland (1987) también es crucial la participación activa de hombres y mujeres ya que la permanencia de la nueva tecnología dependerá de la aceptación de todos y todas sus usuarias. Es más, 
capacitar sólo a los hombres en el uso de determinada tecnología puede producir problemas al interior del grupo doméstico, porque los hombres no necesariamente van a compartir el conocimiento con las mujeres y pueden usarlo como una forma de poder sobre ellas. No olvidemos que la adopción de nuevos conocimientos y el trabajo en grupo también implica crecimiento personal, por lo que no hay que favorecer a un grupo por encima de otro en nuestro trabajo.

Los estudios que utilizan FST desde el enfoque de género traen al centro del análisis la categoría de género, la cual nos permite entender el carácter social y cultural de las relaciones de trabajo y poder al interior del grupo doméstico. Estos estudios han demostrado el papel de las mujeres en las estrategias de supervivencia de familias pobres, y los obstáculos que enfrentan las jefas de familia para sacar adelante a sus hogares. También han ayudado a cuestionar la idea de que los programas dirigidos a hombres automáticamente benefician a todos los y las miembros del grupo doméstico y a las mujeres que lo integran (Poats et al., 1988, p. 4-6).

Para concluir esta sección, quisiéramos resaltar dos puntos principales. Primero, es importante la visión del sistema agrícola como un todo cuyas partes se relacionan entre sí. En este sentido, tenemos que ver la forma en que se relacionan la milpa, el acahual, el solar y el monte. Pero también es necesario analizar el papel de hombres y mujeres en estos espacios, su acceso y control sobre recursos, y los beneficios derivados de las actividades realizadas. No debemos asumir que los espacios públicos (como la milpa) son masculinos y los espacios privados (como el solar) son femeninos, ya que tanto hombres como mujeres desempeñan diversas actividades en cada uno de ellos y es importante que las captemos en su verdadera dimensión. Más que dar una visión descriptiva de la división genérica del trabajo en dichos espacios, tenemos que analizar el valor social que se le asigna al trabajo de hombres y de mujeres y las relaciones de poder que ellos y ellas establecen entre sí. Segundo, al tomar como centro del análisis al grupo doméstico, podremos observar las diferencias en responsabilidad, acceso, control y beneficios por género y edad, así como las implicaciones de estas diferencias para el bienestar de las mujeres.

\section{Conclusiones}

En este trabajo hemos estudiado los aportes de la perspectiva de género para el análisis del manejo de recursos naturales, desde tres ángulos diferentes. Después de presentar brevemente en qué consiste el enfoque de género, expusimos la visión neo-liberal del desarrollo sustentable, que hoy en día se ha convertido en política pública en nuestros países, y la contrastamos con la propuesta desde la perspectiva de género. Concluimos que esta última es más amplia por incluir no sólo aspectos económicos sino también aquellos relacionados con las desigualdades sociales y de género y la participación ciudadana en el proceso de toma de decisiones.

Seguimos con la discusión de dos corrientes teóricas que estudian la relación que establecen hombres y mujeres con el medio ambiente, que son Mujeres y Medio Ambiente y Género y Medio Ambiente. En esta sección dijimos que la segunda es más pertinente porque incluye tanto a hombres como a mujeres en el análisis, sin dejar de remarcar que la participación de estas últimas en proyectos de conservación debe implicar no sólo mejoras para el medio ambiente, sino también para su calidad de vida y posición como mujeres.

Por último, hablamos de los planteamientos y metodología de la Teoría de Sistemas Agrícolas y expusimos su relevancia para el análisis de género. Esta teoría puede ayudarnos a entender mejor las necesidades de determinado grupo social y las formas en que sus procesos de trabajo y calidad de vida pueden ser apoyados y fortalecidos, si incluimos en el análisis las actividades reproductivas y si somos capaces de superar la visión del grupo doméstico como homogéneo y unitario. El trabajo en campo es la herramienta que nos ayudará a y pulir planteamientos y redefinir prioridades en este sentido. 


\section{REFERENCIAS}

BARRÓN, Luis; Alain de REMES (eds.). Crecer y conservar. Definiciones para una política ecológica. México: Cal y Arena. 1996.

BHASIN, Kamila. "Algunas reflexiones sobre el desarrollo y el desarrollo sustentable". En: Isis Internacional. Despejando horizontes. Mujeres en el medio ambiente. Santiago de Chile: Ediciones de las mujeres n. 13, 1993.

BOSERUP, Esther. Women's Role in Economic Development. London: George Allen y Unwin, 1970.

BRAIDOTTI, Rosi. Women, the Environment and Sustainable Development: Towards a Theoretical Synthesis. London: Zed Books, 1994.

CAREAGA, Gloria; Carolina MARTÍNEZ; Leticia MERINO. Construyendo relaciones entre los campos de salud reproductiva y desarrollo sustentable. Segunda reunión del diálogo entre salud reproductiva y desarrollo sustentable, Amatlán, Morelos, 8 y 9 de diciembre de 1997.

CAZÉS, Daniel. La perspectiva de género. Guía para diseñar, poner en marcha, dar seguimiento y evaluar proyectos de investigación y acciones públicas y civiles. México: CONAPO, 1998.

GONZÁLEZ AMADOR, Roberto. "Persistente crecimiento de la miseria en México, indica el Banco Mundial", en La Jornada, 19 de septiembre de 1999.

GUDYNAS, Eduardo. "Los múltiples verdes del ambientalismo latinoamericano", in Nueva Sociedad, v.122, p. 104-115, 1992.

HÄUSLER, Sabine. "Women and the Politics of Sustainable Development", en: Wendy Harcout (coord). Feminist Perspectives on Sustainable Development. Londres: Zed Books, 1994.

JAAKONKARI, Mark. "Análisis de la relación entre neoliberalismo y Desarrollo Sustentable". En: René Valdiviezo Sandoval y Sergio Flores González (coord). Importancia y perspectiva del Desarrollo Sustentable en México. México: Universidad Autónoma de Tlaxcala, 1996. LAGARDE, Marcela. Género y feminismo. Desarrollo humano y democracia. Madrid: Horas, 1997.

LAMAS, Marta. "Introducción", en El género: la construcción cultural de la diferencia sexual. México: PUEGUNAM y Porrúa, 1996.
LEACH, Melissa; Susan JOEKES; Cathy GREEN. Editorial: Gender Relations and Environmental Change" in International Development Studies Bulletin, v. 26, n. 1, p. 1-8, 1995.

LEIS, Héctor Ricardo. "El rol educativo del ambientalismo en la política mundial", Nueva Sociedad, v. 122, p. 116127, 1992.

MÁRQUEZ AYALA, David. "Reporte Económico. América Latina en el 2000, según la CEPAL", en $L a$ Jornada, 17 de enero de 2000.

MIES, Maria. "The global is in the local". En: Caroline Merchant. Terra Femina. Brasil: Redeh \& IDAC, 1992.

POATS, Susan V.; Marianne SCHMINK; Anita SPRING (eds.). Gender Issues in Farming Systems Research and Extension. Colorado: Westview Press, 1988.

RICO, María Nieves. "Género, medio ambiente y sustentabilidad del desarrollo", ponencia presentada en la Séptima Conferencia Regional sobre la Integración de la Mujer en el Desarrollo Económico y Social de América Latina y el Caribe, Santiago de Chile, 19-21 de noviembre de 1997.

RODDA, Annabel. Women and the Environment. Londres: Zed books, 1993.

SHANER, W. W.; P. F. PHILIPP; W. R. SCHMEHL. Farming Systems Research and Development. Guidelines for developing Countries. Colorado: Westview Press, 1981. SUTHERLAND, Alistair. Sociology in Farming Systems Research. Londres: Overseas Development Institute, 1987. VÁZQUEZ GARCÍA, Verónica. "Género, medio ambiente y desarrollo sustentable. Reflexiones teóricas y metodológicas", en V. Vázquez García (coord.), Género, sustentabilidad y cambio social en el México rural. Texcoco: Colegio de Postgraduados. 1999.

VÁZQUEZ GARCÍA, Verónica. "Coffee Production and Household Dynamics. The popolucas of Ocotal Grande, Veracruz", in Agriculture an Human Values 2001. En prensa.

WARRING, Marilyn. If Women Counted. A New Feminist Economics. Londres: Macmillan Press, 1988. 